International Journal on Cybernetics \& Informatics (IJCI) Vol. 5, No. 2, April 2016

\title{
SVD AUDIO WATERMARKING
}

\author{
Veena Gopan ${ }^{1}$ and Mary Joseph ${ }^{2}$ \\ ${ }^{1}$ Department of Electronics and Communication, Mar Athanasius College Of \\ Engineering, A.P.J Abdul Kalam Technological University, Kerala, India \\ ${ }^{2}$ Associate Professor, Department of Electronics \&Communication Engineering \\ M.A.College of Engineering, Kothamangalam
}

\begin{abstract}
It proposes an approach for audio watermarking using the singular value decomposition (SVD) mathematical technique. After transforming it into a 2-D format the encrypted image is embedded in the singular values of the audio signal. After watermark embedding, the audio signal is transformed again into a 1-D format. For encrypt the image chaotic encryption is used. It improves the quality of extracted images as proved experimentally, where it resists the noise and different attacks.
\end{abstract}

\section{KEYWORDS}

SVD, watermarking, baker mapping, chaotic encryption, decryption, SVs (Singular Values)

\section{INTRODUCTION}

Digital Watermarking has found many applications in image, video and audio transmission. In audio watermarking algorithms most of them are designed to achieve an efficient detection of the watermark without extracting meaningful information from the watermarked audio signal designed to achieve an efficient detection of the watermark without extracting meaningful information from the watermarked audio signal [8-9]. There is a need for a robust audio watermarking approach with a higher degree of security, which can be achieved by embedding encrypted images in audio signals [8-9]. In this project, the chaotic Baker map is used for the encryption of the watermark image [1-3]. Then, the watermark is embedded in the audio signal using the SVD mathematical technique [1]. The audio signal is transformed into a 2-D format .The singular values (SVs) of the resulting 2-D matrix are used for watermark embedding. From the speech signal at the receiver end the water mark is extracted and then the watermark is decrypted to get the message.

\section{SYSTEM IMPLEMENTATION}

In this project the cover speech signal is converted to 2-D for SVD water marking, then the message to be transmitted is encrypted by using baker mapping version A [1-3], and watermarked the message into the 2-D changed cover signal using SVD technique [1]. Then for transmission it is again converted to $1 \mathrm{D}$.

DOI: $10.5121 /$ ijci.2016.5240 
At the receiver end the 1-D matrix is changed to 2-D for extracting watermark. The extracted watermark is decrypted to get the message, and then the result is compared with the original message. The block diagram is given in fig:2.1

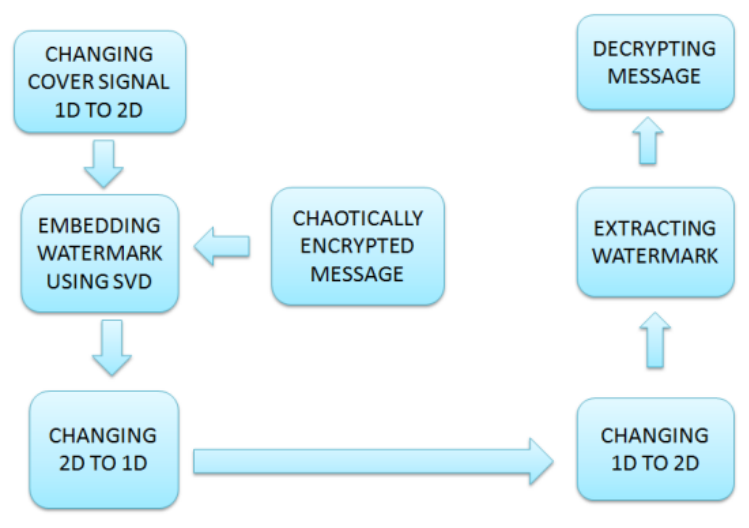

Fig 2.1 block diagram of system

To find out its response with noise, noise is added with the transmitted signal and the received message is compared with the original message using different signal to noise ratio.

\subsection{SVD (SINGULAR VALUE DECOMPOSITION)}

The Singular Value Decomposition decomposes the cover signal into 3 matrices each of size same as that of cover 2-D matrix [1].

$$
\operatorname{SVD}(\text { Cover signal })=\text { U S V' }
$$

Its singular value diagonal matrix is $\mathrm{S}$ of the cover signal, the encrypted message matrix is watermarked into the matrix $\mathrm{S}$ using a constant $\mathrm{k}=0.01$, which makes the signal undistorted. And the watermarked matrix is again converted to 1-D for transmission.

\subsection{BAKER MAPPING (ENCRYPTION)}

Baker mapping encrypt the message block by block, which will be easy for decryption [2,3]. Here we uses version A baker mapping of 8X8 matrix. Version A baker mapping can be done by using 5 different keys. The key elements are selected according the following considerations

- The elements sum must be 8

- 1 is not taken as element

- 8/each element must be perfectly divisible The steps for baker mapping are given below

- Take $8 * 8$ message matrix

- Encrypt the message using the equation $\mathrm{B}\left(\mathrm{x}^{\prime}, \mathrm{y}^{\prime}\right)=\mathrm{A}\left(\frac{\mathrm{N}}{\mathrm{n}_{\mathrm{i}}}\left(\mathrm{x}-\mathrm{N}_{\mathrm{i}}\right)+\mathrm{ymod} \frac{\mathrm{N}}{\mathrm{n}_{\mathrm{i}}}, \frac{\mathrm{n}_{\mathrm{i}}}{\mathrm{N}}\left(\mathrm{y}-\mathrm{y} \bmod \frac{N}{n_{i}}\right)+N_{\mathrm{i}}\right)$, Where $\mathrm{N}=8$.

- $\quad B$ will be the baker mapped message 


\subsection{EMBEDDING WATERMARK USING SVD}

The flowchart for embedding watermark is given in the figure 2.2. The audio signal is transformed into a 2-D format and the singular values (SVs) of the resulting 2-D matrix are used for watermark embedding. The chaotically encrypted message is embedded into the cover matrix using SVD method. And the 2-D matrix is transformed to 1-D

The steps for embedding a watermark using SVD method are given below

- The 1- D audio signal is transformed into a 2-D matrix (A matrix).

The SVD is performed on the A matrix.

$$
\mathrm{A}=\mathrm{US} \mathrm{V}^{\mathrm{T}}
$$

- The watermark (W matrix) is added to the SVs of the original matrix.

$$
\mathrm{D}=\mathrm{S}+\mathrm{KW}
$$

- A small value of $\mathrm{K}$ of about 0.01 is required to keep the audio signal undistorted. The SVD is performed on the new modified matrix (D matrix

$$
\mathrm{D}=\mathrm{U}_{\mathrm{W}} \mathrm{S}_{\mathrm{W}} \mathrm{V}_{\mathrm{W}}^{\mathrm{T}}
$$

- The watermarked signal in 2-D format (Aw matrix) is obtained using the modified matrix of SVs (Sw matrix).

$$
\mathrm{A}_{\mathrm{W}}=\mathrm{US}_{\mathrm{W}} \mathrm{V}^{\mathrm{T}}
$$

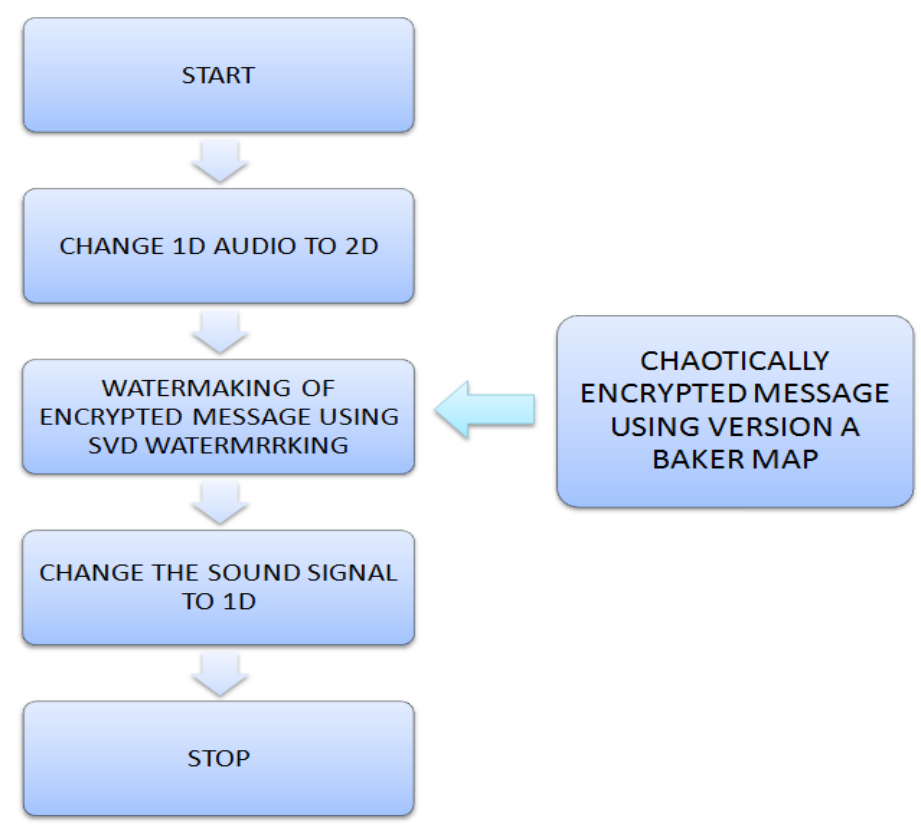

Fig:2.2 flowchart for embedding watermark 


\subsection{EXTRACTING WATERMARK}

The flowchart for extracting watermark is given in the figure 2.3.The received 1-D is transformed to 2-D and the watermark is extracted. The watermark is decrypted to get the original message [13].

The steps for extracting watermark are

- The 2-D Aw matrix is transformed again into a 1-D audio signal.

- $\quad$ The SVD is performed on the possibly distorted watermarked image ( $\mathrm{A}^{*} \mathrm{~W}$ matrix).

$$
\mathrm{A}_{\mathrm{W}}^{*}=\mathrm{U}^{*} \mathrm{~S}_{\mathrm{W}}^{*} \mathrm{~V}^{* \mathrm{~T}}
$$

- $\quad$ The matrix that includes the watermark is computed.

$$
\mathrm{D}^{*}=\mathrm{U}_{\mathrm{W}} \mathrm{S}_{\mathrm{W}}^{*} \mathrm{~V}_{\mathrm{W}}^{\mathrm{T}}
$$

- The possibly corrupted encrypted watermark is obtained.

$$
\mathrm{W}^{*}=\left(\mathrm{D}^{*}-\mathrm{S}\right) / \mathrm{K}
$$

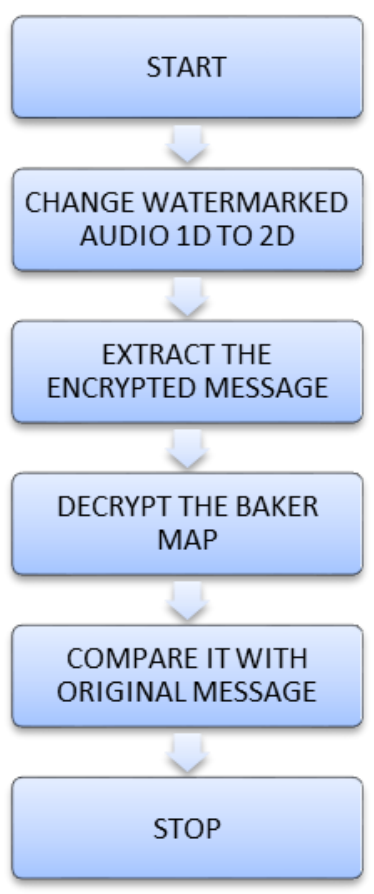

Fig.2.3 flowchart for extracting watermark

\subsection{DECRYPTION OF BAKER MAP}

- $\quad$ Take L=B' 
International Journal on Cybernetics \& Informatics (IJCI) Vol. 5, No. 2, April 2016

- Use the following equation for decryption

$\left.\mathrm{K}\left(\mathrm{x}^{\prime}, \mathrm{y}^{\prime}\right)=\mathrm{L}\left(\frac{\mathrm{N}}{\mathrm{n}_{\mathrm{i}}}\left(\mathrm{x}-\mathrm{N}_{\mathrm{i}}\right)+\mathrm{y} \bmod \frac{\mathrm{N}}{\mathrm{n}_{\mathrm{i}}}, \frac{\mathrm{n}_{\mathrm{i}}}{\mathrm{N}}\left(\mathrm{y}-\mathrm{y} \bmod \frac{N}{n_{i}}\right)+N_{\mathrm{i}}\right)\right)$, Where $\mathrm{N}=8$,

- Take $\mathrm{E}=\mathrm{K}$, then $\mathrm{E}$ will be the decrypted message

The message before baker mapping is given in the fig:2.4 and after baker mapping is given in the fig :2.5.Then change the message matrix with the baker mapped matrix for embedding.

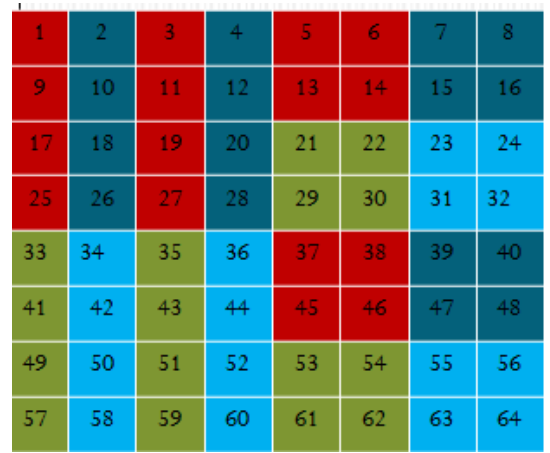

Fig: 2.4 message before baker mapping

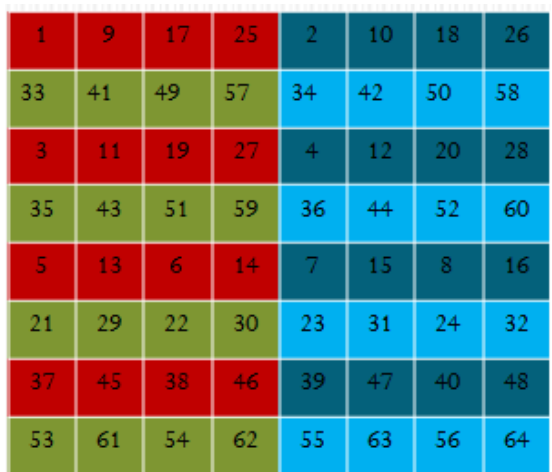

Fig: 2.5 message after baker mapping using a key

\section{RESULTS}

Matlab software is used for simulation of the system which is explained in chapter 2. The cover signal required for system is $3 \times 256 \times 256$ elements for transmission. For decryption we need U matrix and V matrix.256 × $256 \mathrm{U}$ and $256 \times 256 \mathrm{~V}$ is the watermarked matrix after SVD decomposition. They are embedded in to the cover signal. The encrypted matrix is embedded using SVD technique. The $\mathrm{U}$ and $\mathrm{V}$ matrix elements multiplied with 0.1 and added respectively with the elements of the cover signal. The cover signal is a sound signal and waveform of original sound is given in fig:3.1. 
International Journal on Cybernetics \& Informatics (IJCI) Vol. 5, No. 2, April 2016

The message is of $8 \times 8$ size which is to be encrypted using baker mapping and watermarked using SVD to the above cover signal. The message example used is given in Fig: 3.2.The message is encrypted using baker mapping technique and the result will be like in figure fig:3.3 Then the encrypted message is watermarked into the cover signal using SVD method. And the U and $\mathrm{V}$ matrices are also added to the cover signal for decryption purpose. The resultant waveform of the water marked speech signal is given Fig: 3.4. The speech after watermarking makes no difference when played. And the waveforms also differ a little, which is hardly detectable. The extracted encrypted message from the watermarked sound wave is given in fig:3.5.

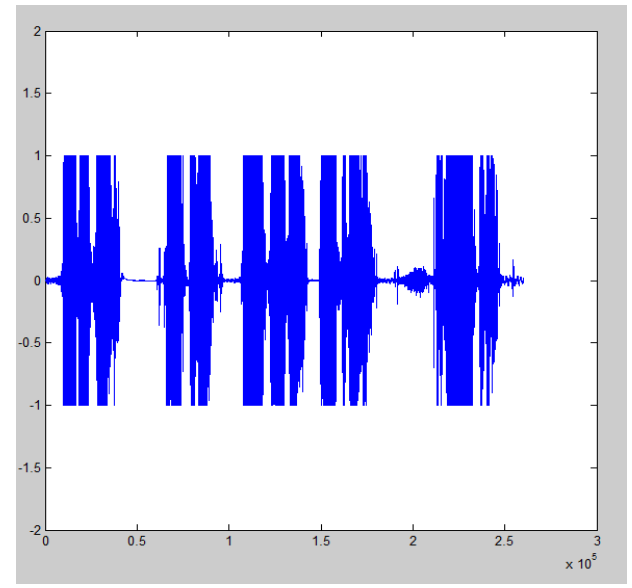

Fig: 3.1 waveform of original sound signal

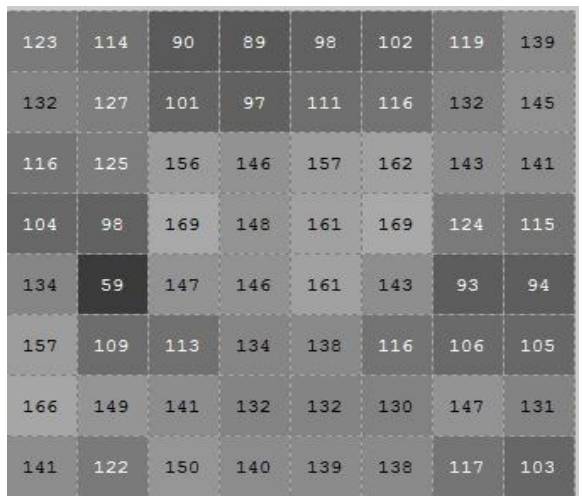

Fig: 3.2 message 
International Journal on Cybernetics \& Informatics (IJCI) Vol. 5, No. 2, April 2016

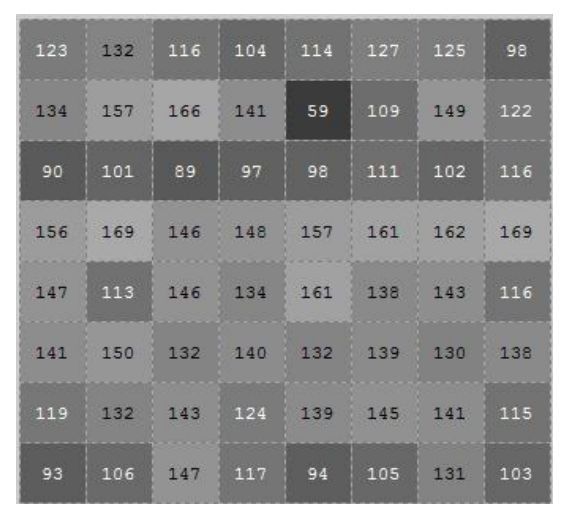

Fig: 3.3 encrypted message

The $\mathrm{U}$ and $\mathrm{V}$ matrices are extracted from the received speech signal and by using the reverse operation in baker mapping the message is decrypted. The decrypted message is given Fig: 3.6 the decrypted watermark is same as the original message. Then for studying the performance of the system, watermarked speech signal is subjected to noise. And by varying the SNR from 0 to 50 , the performance is evaluated for 64 elements. A graph is plotted verses SNR and error. SNR greater than $45 \mathrm{~dB}$ indicates no error and hence the signal is extracted. The error increases with decreasing SNR. The figure is given in fig:3.7

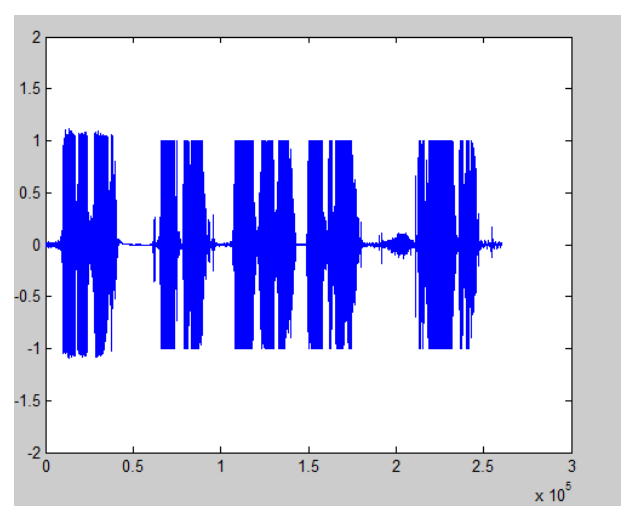

Fig: 3.4 waveform of watermarked audio signal

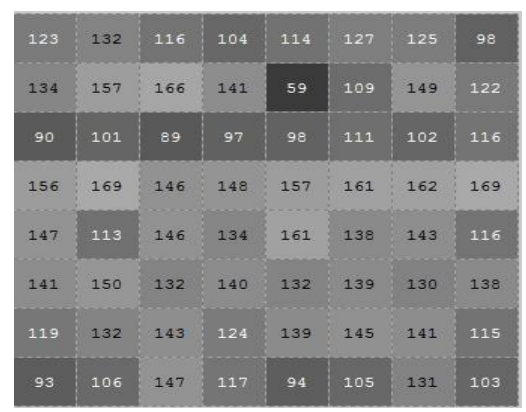

Fig: 3.5 extracted watermark from audio 
International Journal on Cybernetics \& Informatics (IJCI) Vol. 5, No. 2, April 2016

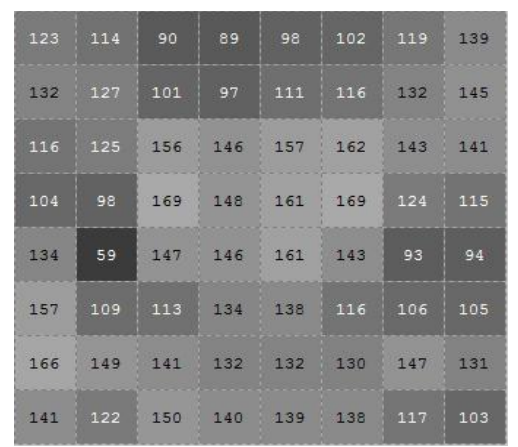

Fig: 3.6 decrypted message

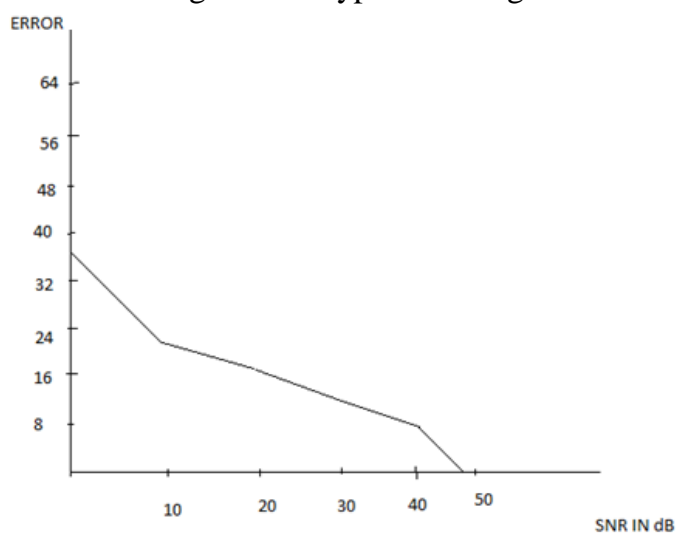

Fig 3.7 graph of SNR in dB against the error

\subsection{ADVANTAGES AND DISADVANTAGES}

The advantages and disadvantages of the SVD audio watermarking method are listed below Advantages

- When $\mathrm{SNR}>45 \mathrm{~dB}$ the received message has no errors.

- The encryption scheme used here is baker mapping which is more secure.

- The keys used for encryption are more secure which reduces Bruce-force guessing attacks.

Disadvantages

- Only 8x8 messages can be watermarked at a time, because encryption using baker mapping can be done for only $8 \mathrm{X} 8$ matrix at a time.

- The message length greater than 64 need to be cut into blocks of 64 elements. 
International Journal on Cybernetics \& Informatics (IJCI) Vol. 5, No. 2, April 2016

\section{CONCLUSION}

This paper has presented an efficient security algorithm for Bluetooth network through SVD audio watermarking approach. In this algorithm, encrypted image is embedded as watermarks in audio signals to achieve a high degree of security. Experimental results have proved that watermark embedding in the proposed approach does not deteriorate the audio signals. It has been clear through experiments that the chaotic Baker map encryption algorithm is an efficient algorithm for watermark encryption.

\section{ACKNOWLEDGEMENTS}

The author takes this opportunity to thank all those who have been directly or indirectly involved in making this project a success. The author express her honest gratitude to the project guide Prof. DAVID SOLOMON GEORGE(Associate Professor, Dept. of ECE, RIT Kottayam) who is also the project co-coordinator, for his constant encouragement, inspiration and enthusiastic guidance and without whose continuous support and patience, it would have been extremely difficult to complete this work. The author expresses her sincere gratitude to all the teachers and staff of Rajiv Gandhi Institute of Technology.

\section{REFERENCE}

[1] M. A. M. El-Bendary, A. Haggag, F. Shawki, and F. E. Abd-El-Samie, "Proposed Approach for Improving Bluetooth Networks Security through SVD Audio Watermarking "2012 IEEE ,pp-594-598

[2] Feng HUANG, Yong FENG," Security analysis of image encryption based on two dimensional chaotic maps and improved algorithm"2009, pp-5-9

[3] Jiri fridrich, "Symmetric ciphers based on two-dimensional chaotic maps"1998, pp-1259-1284

[4] B. Macq, J. Dittmann, and E. J. Delp, "Benchmarking of Image Watermarking Algorithms for Digital Rights Management", Proceedings of The IEEE, Vol. 92, No. 6, pp. 971-984, 2004.

[5] Z. M. Lu, D. G. Xu, and S. H. Sun, "Multipurpose Image Watermarking Algorithm Based on Multistage Vector Quantization" IEEE Transactions on Image Processing, Vol. 14, No. 6, pp. 822 $831,2005$.

[6] H. S. Kim and H. K. Lee, "Invariant Image Watermark Using Zernike Moments", IEEE Transactions on Circuits And Systems For Video Technology, Vol. 13, No. 8, pp. 766-775, 2003.

[7] W. C. Chu, " DCT-Based Image Watermarking Using Subsampling", IEEE Transactions on Multimedia, Vol. 5, No. 1, pp.34-38, 2003.

[8] L. Ghouti, A. Bouridane, M. K. Ibrahim, and Said Boussakta, "Digital Image Watermarking Using Balanced Multiwavelets", IEEE Transactions on Signal Processing, Vol. 54, No. 4,pp. 1519-1536, 2006.

[9] S. Xiang and J. Huang, "Histogram-Based Audio Watermarking Against Time-Scale Modification and Cropping Attacks", IEEE Transactions on Multimedia, Vol. 9, No. 7, pp. 1357-1372, 2007.

[10] Z. Liu and A. Inoue, "Audio Watermarking Techniques Using Sinusoidal Patterns Based on Pseudorandom Sequences", IEEE Transactions On Circuits And Systems For Video Technology, Vol. 13, No. 8, pp. 801-812, 2003 
International Journal on Cybernetics \& Informatics (IJCI) Vol. 5, No. 2, April 2016

\section{AUTHOR}

VeenaGopan received B-tech graduation from $\mathrm{M}$ G University in Electronics and Communication engineering. Now pursuing M-tech from A P J Abdul Kalam Technological University in VLSI and Embedded System.

Mary Joseph received M.Tech Degree in Microwave and Radar from Cochin University of Science and Technology (CUSAT), Kochi, India, in 1997. Currently she is working as Associate Professor in M. A. College of Engineering, Kothamangalam. She has joined in M. A. College of Engineering in 1991 as Assistant Professor. In between she worked at Birla Institute of Science \& Technology-Pilani's (BITS-PILANI) Dubai Campus for 9 years as Assistant Professor during 2000-2008. Her Research interests include Microstrip

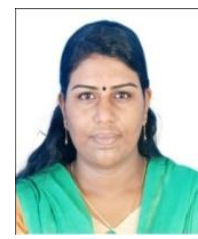
Antennas and Uniplanar Antennas. 